\section{Massive pleural effusion due to IgG multiple myeloma}

\author{
Kathryn J. Lang,' Surjit Lidder, \\ Robin Aitchison'
}

'Department of Haematology, Wycombe General Hospital, UK; ${ }^{2}$ Department of Trauma and Orthopaedics, Royal London Hospital, Whitechapel, London

\begin{abstract}
Pleural effusion directly attributable to multiple myeloma is exceedingly uncommon and is said to occur in only $1-2 \%$ of cases. Of these around $80 \%$ occur in IgA disease. We report a case of myelomatous pleural effusion (proven on cytological and immunohistochemical analysis) in a patient with the IgG- $\kappa$ subtype. We describe the diagnosis, pathogenesis and management of this condition and show the radiological and cytological evidence of the case.
\end{abstract}

\section{Introduction}

Multiple myeloma is a clonal B-cell malignancy characterized by proliferation of plasma cells accumulating mainly in the bone marrow and secreting paraprotein. ${ }^{1}$ Myeloma accounts for around 1\% of all malignancies and UK national statistics for 2006 report an incidence of 6.4 cases per 100,000 annually. ${ }^{2}$ Mean age at diagnosis is 65 years.

Pleural effusion directly attributable to myeloma is exceedingly rare (1-2\% of cases). ${ }^{3}$ We present a case of pleural effusion arising rapidly in a patient with long-standing multiple myeloma and found to be a myelomatous effusion.

\section{Case Report}

A 74-year old man with longstanding IgG kappa myeloma presented with a two week history of increasing dyspnoea on exertion. Blood tests showed a normocytic anemia (Hb: 7.4 $\mathrm{g} / \mathrm{dL}, \mathrm{MCV}: 90.1 \mathrm{fl}$ ), and renal impairment (urea: $9.2 \mathrm{mmol} / \mathrm{L}$, creatinine: $248 \mu \mathrm{mol} / \mathrm{L}$ ). Serum electrophoresis suggested rapid disease progression with an increase in paraprotein from $14 \mathrm{~g} / \mathrm{L}$ to $50 \mathrm{~g} / \mathrm{L}$ over a six week period. Chest radiographs showed a large left sided pleural effusion (Figure 1). Diagnostic tap of the effusion produced heavily blood stained fluid with features suggestive of an exudate (pH: 7.62, total protein: 93 g/L, LDH: $506 \mathrm{U} / \mathrm{L}$ ).
The effusion persisted, necessitating therapeutic drainage which produced four liters of bloodstained fluid. Computed tomography (CT) of the chest was radiologically suggestive of mesothelioma (Figure 2) but cytological analysis of the pleural fluid showed plasma cells consistent with myeloma (Figure 3).

Subsequent immunohistochemistry revealed $\kappa$ light chain restriction and VS38 positivity. A diagnosis of pleural effusion secondary to multiple myeloma was made and the patient was commenced on bortezomib and dexamethasone therapy seven days after first presenting. No further pleural aspiration was necessary from this point and repeat chest radiography (Figure 4) taken seven days after starting treatment showed resolving pleural disease and the patient reported improved symptomatology.

\section{Discussion}

Pleural effusion is uncommon in multiple myeloma; it has been suggested that it occurs in approximately $6 \%$ of cases. Most of these are not directly due to myelomatous infiltra-

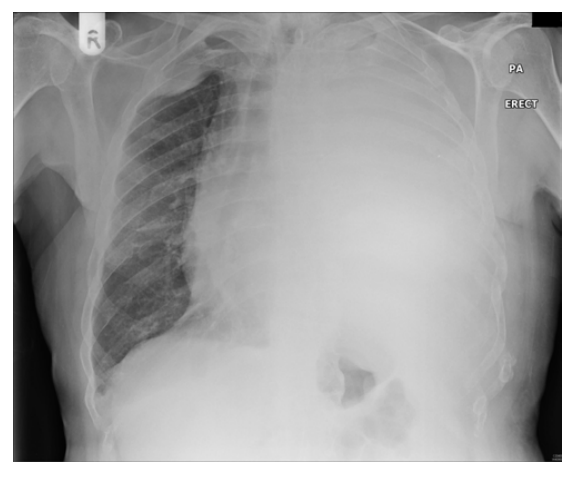

Figure 1. Chest radiographs showed a large left sided pleural effusion.

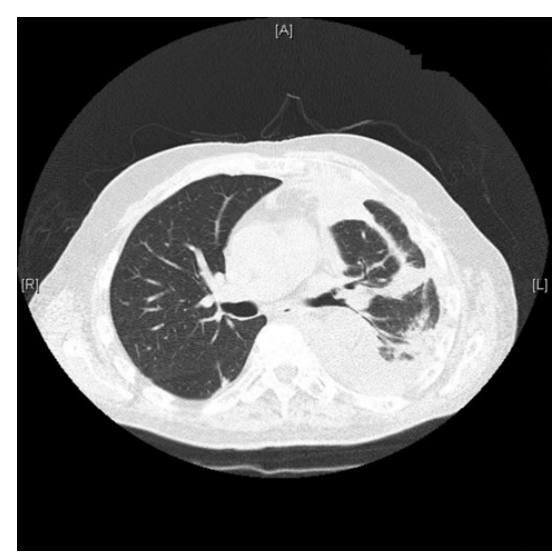

Figure 2. Computed tomography of the chest was radiologically suggestive of mesothelioma.
Correspondence: Kathryn J. Lang, Department of Haematology, Wycombe General Hospital, UK.

E-mail: K.J.Lang@doctors.org.uk

Key words: hematology, multiple myeloma, pleural effusion.

Received for publication: 1 0ctober 2009. Revision received: 21 October 2009.

Accepted for publication: 22 October 2009.

This work is licensed under a Creative Commons Attribution 3.0 License (by-nc 3.0).

(C) Copyright K.J. Lang et al., 2009

Licensee PAGEPress, Italy

Hematology Reviews 2009; 1:e18

doi:10.4081/hr.2009.e18

tion but to related pathologies such as pulmonary embolus, heart failure and nephrotic syndrome. Effusion directly due to myeloma is said to occur in less than $1 \%$ of cases with approximately $80 \%$ of these being in IgA disease. $^{3}$

Involvement of the cavities is an ominous feature in multiple myeloma and thought to represent either a late manifestation in the

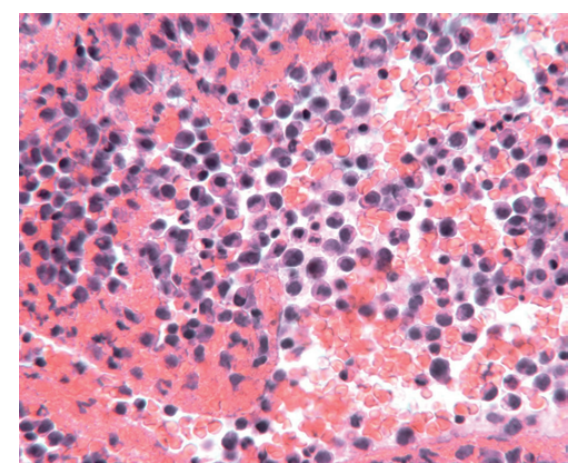

Figure 3. Cytological analysis of the pleural fluid showed plasma cells consistent with myeloma.

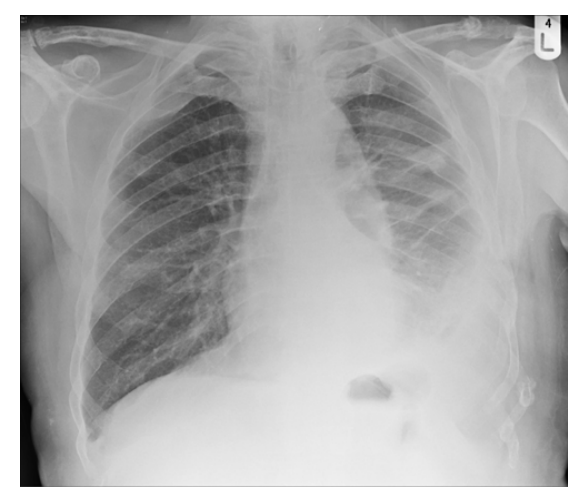

Figure 4. Repeat chest radiography taken seven days after starting treatment showed resolving pleural disease. 
natural history of the disease or a feature of the aggressive behavior of myeloma. Diagnosis is based on cytological analysis and immunohistochemical staining, pleural biopsy may also yield a diagnosis of myeloma. Malignant plasma cells within the cytological preparation show a typical basophilic cytoplasm with large, eccentric nuclei and prominent nucleoli. ${ }^{4}$

Several possible mechanisms are postulated for myelomatous pleural effusion: invasion from adjacent skeletal lesions; extension from chest wall plasmacytomas; tumor infiltration of the pleura; and mediastinal lymph node involvement causing lymphatic obstruction., Currently, the exact mechanism of pathogenesis remains unknown.

The management is two-stage. First, the effects of the effusion must be alleviated with therapeutic and diagnostic thoracocentesis. Once myelomatous pleural effusion is confirmed on cytology, and/or immunohistochemistry, systemic chemotherapy is required to slow the advance of the disease. There is as yet no consensus as to how best to manage this rapid and aggressive phase of disease but we anecdotally show evidence of a good response to bortezomib and dexamethasone therapy.

We suggest that this case highlights the vital importance of diagnostic clarity, where possible, in finding the underlying etiology of a pleural effusion. Myelomatous pleural effusion carries a poorer prognosis and more work is needed to understand the exact pathogenesis of this condition.

\section{References}

1. Provan D, Singer CRJ, Baglin T, Lilleyman J. Oxford Handbook of Clinical Haematology, 2nd ed. Oxford University Press, 2004.

2. UK National Cancer Statistics 2006, Cancer Research UK. http://info.cancerresearchuk.org/cancerstats/types/multiplemyeloma/incidence/ Accessed 01/10/2009.

3. Shirdel A, Attaran D, Ghobadi H, Ghiasi T. Myelomatous pleural effusion. Tanaffos, 2007;6:68-72.

4. Gogia A, Agarwal PK, Jain S, Jain KP. Myelomatous pleural effusion. J Assoc Phys India, 2005;5:734-6.

5. Yu FC, Chang JY, Hwang SH, et al. Kappalight chain multiple myeloma with myelomatous pleural effusion: Case report and review of the literature. J Med Sci 1994;15: 49-56. 